\title{
A CONTRIBUIÇÃO DA ORGANIZAÇÃO E DA VISUALIZAÇÃO DA INFORMAÇÃO PARA OS SISTEMAS DE RECUPERAÇÃO DE INFORMAÇÃO
}

\author{
LA CONTRIBUCIÓN DE LA ORGANIZACIÓN Y LA \\ VISUALIZACIÓN DE INFORMACIÓN PARA SISTEMAS DE \\ RECUPERACIÓN DE INFORMACIÓN
}

\begin{abstract}
Jessica Monique de Lira Vieira - jessicalirav@yahoo.com.br Mestre em Ciência da Informação pelo Programa de Pós-Graduação em Ciência da Informação da Universidade Federal de Pernambuco (UFPE).

Fabio Assis Pinho - fabiopinho@ufpe.br Doutor em Ciência da Informação. Professor no Departamento de Ciência da Informação e no Programa de Pós-Graduação em Ciência da Informação da UFPE.
\end{abstract}

\section{RESUMO}

Introdução: Buscando propor estratégias que potencializem o entendimento da memória armazenada em Sistemas de Recuperação de Informação (SRIs), considera-se, neste trabalho, como alternativa, a adoção de procedimentos de Organização da Informação (OI) e Visualização da Informação (VI).

Objetivo: Nesse contexto, a pesquisa tem como objetivo analisar e identificar a relação entre os aspectos da OI e da VI do ponto de vista da contribuição das duas áreas para os SRIs, de maneira a potencializar o entendimento da memória por eles armazenada, razão pela qual a pesquisa é caracterizada como exploratória e bibliográfica.

Metodologia: O percurso metodológico utilizado para realização da pesquisa consiste em levantamento bibliográfico, para obtenção do embasamento científico para melhor compreensão e análise de cada área; levantamento de sistemas que fazem uso de interface de VI em sua estrutura, bem como da análise dos elementos de Ol necessários para organização e representação de conteúdos a serem transmitidos em estruturas visuais.

Resultados: A partir disso, evidenciou-se a interligação entre as áreas abordadas, quando aplicadas em sistemas que lidam com a recuperação da informação. 
Conclusões: Desse modo, concluiu-se que a Ol e a VI podem alcançar promissores resultados se atuarem em parceria para otimização na recuperação da memória armazenada em SRls.

Palavras-chave: Produção científica. Reprodução. Distinção. Ciência da Informação.

\section{INTRODUÇÃO}

A crescente produção de informações, juntamente com os avanços tecnológicos, desempenha um papel fundamental na consolidação da sociedade atual, enquanto "sociedade da informação". Sociedade em que a informação é vista, como afirma Le Coadic (2004), como uma necessidade fundamental, ou seja, como uma necessidade vital para o desenvolvimento de, praticamente, todas as atividades humanas. É nesse cenário, no qual a informação, dotada de tamanho valor, enfrenta um crescimento na sua produção e disponibilização, que se faz necessária a busca de métodos adequados para, significativamente, gerenciar a quantidade de informação disponível de forma a possibilitar seu acesso e uso.

Diante desse contexto e da necessidade de proporcionar o acesso para posterior uso da informação, torna-se salutar propor soluções para os problemas relacionados à informação e à sua recuperação. Nesse âmbito, uma das áreas preocupadas e envolvidas com essa questão é a Ciência da Informação (Cl) que, juntamente com outras áreas, é uma participante ativa e deliberada na evolução da sociedade da informação (SARACEVIC, 1995); e, como aponta Dias (2001), tem como questão básica o acesso à informação, ou a facilitação desse acesso, o que constitui uma das razões gerais de sua existência.

Nessa perspectiva, entre as áreas estudadas pela $\mathrm{Cl}$, está a Recuperação da Informação $(\mathrm{Rl})$, que preocupa-se em disponibilizar a informação para satisfação de uma necessidade do usuário. Diante disso, torna-se oportuno evidenciar o papel dos sistemas que atuam no intuito de tornar possível, a partir de um universo de documentos disponíveis, a recuperação daqueles que venham a ser úteis para quem deles necessita, os chamados Sistemas de Recuperação de Informação (SRls), cujo objetivo, de forma genérica, é facilitar o acesso e possibilitar a recuperação e o entendimento da memória produzida e por eles armazenada. Tais sistemas, como 
destaca Araújo (1995), constituem a "memória humana registrada."

No entanto, para que os SRIs atuem de maneira satisfatória, é fundamental que haja uma atenção maior no que tange à organização das informações armazenadas por esses sistemas, pois, como destaca Brascher e Carlan (2010), em um sistema de informação, a qualidade obtida na recuperação da informação depende, substancialmente, dos procedimentos e instrumentos utilizados para organização da informação.

Nesse âmbito, tem-se a Organização da Informação (OI), que exerce uma atividade nuclear enquanto mediadora, sendo responsável por estabelecer uma ponte entre a representação e o uso da informação, caracterizando, assim, seu papel, quando se lida com objetos informacionais. Nesse sentido, a Ol tem como função, resumidamente, organizar registros de informação para arranjá-los sistematicamente, de forma a possibilitar sua recuperação e posterior uso.

Para desenvolvimento de suas práticas, a Ol fundamenta-se em um aspecto essencial, o tratamento da informação. O tratamento da informação, de acordo com Pinto e Molina (1993 apud DAL' EVEDOVE, 2011), consiste no processo de gerar representações documentais, as quais, inseridas no contexto de sistemas de informação, atuam como instrumentos de busca, cujo propósito corresponde à facilitação da consulta e disseminação do documento primário.

Considerando essa realidade, ressalta-se o importante valor que a Ol representa para os sistemas de informação, tendo em vista que a adequada organização tem o poder de interferir diretamente na recuperação de informações precisas e que, em contrapartida, a informação sem uma organização prévia pode permanecer oculta e sem uso. Reafirmando essa ideia, Svenonius (2000) enfatiza, ainda, que a eficácia de um sistema que lida com a recuperação da informação está diretamente relacionada à inteligência adotada para a sua organização.

Outro ponto a ser tratado quando se lida com a otimização na recuperação e uso da memória armazenada nos sistemas de informação refere-se a Visualização da Informação (VI). A VI pode ser vista como uma área que tem como finalidade potencializar a apropriação de informação, por meio de recursos gráficos conhecidos como estruturas de visualização da informação (DIAS; CARVALHO, 2007).

Nesse sentido, a VI apóia-se na representação visual como maneira de auxiliar a compreensão de informações armazenadas e que foram, previamente, 
organizadas, objetivando facilitar a localização e recuperação eficaz de itens que atendam a uma necessidade. Para isso, a VI faz uso de interfaces gráficas que possibilitam a interação do usuário com os sistemas, por meio de ambiente de pesquisa agradável, com a finalidade de maximizar o uso da informação.

No entanto, para que a VI possa exercer sua função de forma satisfatória, é preciso que os procedimentos de Ol ofereçam um suporte que torne possível a adoção de representações visuais pelos sistemas (ZHANG, 2008), pois a Ol se configura como área fundamental na organização e tratamento da informação, e que afeta diretamente sua recuperação; por outro lado, a VI se configura como uma poderosa aliada, capaz de potencializar o acesso, localização e recuperação da informação organizada. Assim, tem-se a Ol como um processo interno aos sistemas de recuperação de informação, porém, muitas vezes, não transparente; e a VI que, atuando mediante representação visual, visa à facilitação na apropriação das informações previamente estruturadas e organizadas.

É nesse cenário que se estabelece o objetivo deste trabalho, isto é, analisar a relação entre os aspectos da Ol e da $\mathrm{VI}$ do ponto de vista da contribuição das duas áreas para os SRIs, de maneira a potencializar o entendimento da memória por eles armazenada.

\section{RECUPERAÇÃO DA INFORMAÇÃO}

A necessidade de informação é vista, segundo Le Coadic (2004), como uma necessidade física fundamental, ou seja, necessita-se de informação para construir, solucionar, preencher lacunas, esclarecer, informar e formar o ser humano desde as meras atividades cotidianas. Sendo assim, é nesse contexto que se insere a Rl, como área que se preocupa com o uso da informação para satisfação de uma necessidade informacional, visando tornar acessível a informação para quem dela necessita. Diante de tal responsabilidade, a RI pode ser considerada uma das áreas essenciais no campo da Ciência da Informação. Como explicitou Saracevic (1996), a Recuperação da Informação é o maior ramo da Cl.

Adequando a recuperação da informação à realidade atual, fortemente influenciada pelo intensivo uso de tecnologias de comunicação e informação, que tem transformado o novo século em um mundo cada vez mais ligado em rede de informação global, em que milhões de pessoas se conectam produzindo e 
recuperando informação em tempo real, faz surgir novas formas de pensar, interagir e viver. Esse desenvolvimento trouxe como consequências da explosão da informação, caracterizada pelo aumento da quantidade de informações produzidas e armazenadas, a diversidade e possibilidade de reprodução sem limites (FURTADO, 2010).

Neste universo intensivo de tecnologias de informação e comunicação, e em meio a essa gama de informações, os sistemas eletrônicos, que hoje permeiam e mesmo viabilizam virtualmente quase todas as atividades humanas, objetivam organizar, armazenar e possibilitar o acesso às informações neles existentes, contribuindo para a disseminação da informação e preservação da memória que, segundo Fragoso (2009), é um patrimônio cultural da sociedade e é responsável por alimentar a história da nação.

Nesse contexto, os sistemas que trabalham para tornar a recuperação possível são conhecidos como Sistemas de Recuperação de Informação, e têm como propósito tornar acessível o grande volume de documentos produzidos, de maneira que possam ser recuperados e disseminados, possibilitando a recuperação e o entendimento da memória produzida e armazenada neles. Lunardi (2008) destaca, ainda, que o principal objetivo de um SRI é a seleção, dentro de um universo de documentos disponíveis, do conjunto de documentos relevantes para uma necessidade de informação do usuário. Nessa mesma linha de raciocínio, Araújo Junior (2007) afirma que os SRls dizem respeito a um sistema de operações interligadas para identificar, dentre um grande conjunto de informações, aquelas que são, de fato, pertinentes, ou seja, que estão de acordo com a necessidade expressa pelo usuário.

Neste sentido, recuperar informações relevantes que atendam à necessidade do usuário é a função dos SRIs. Para que isso seja possível, se faz necessário um olhar especial para a organização prévia dessas informações, pois, quando se trata de recuperação e de sistemas que lidam com informações e documentos, não se pode deixar de analisar os aspectos inerentes à organização da informação.

\section{ORGANIZAÇÃO DA INFORMAÇÃO}

O verbo "organizar", de acordo com o Dicionário Houaiss, significa "dar determinada ordem a" (série de itens); "dispor de forma ordenada"; "dar forma 
regular às partes de" (um todo); "arrumar, ordenar". Assim também se comporta o ser humano, organizando naturalmente os muitos tipos de coisas em sua vida: os livros em estantes, as roupas em armários, utensílios de cozinha em gavetas, documentos específicos em pastas etc. Essa organização, mesmo que inconscientemente, visa à facilitação de uma futura localização e recuperação dos itens armazenados.

De maneira geral, pode-se dizer que se organiza para poder compreender o mundo e se comunicar melhor. Por exemplo, ao explicar a alguém o significado de determinada palavra, normalmente recorre-se a sua classificação, incluindo a palavra em um grupo maior que a define. Assim, ao explicar o significado da palavra "cadeira", seria dito que é um móvel (classe) que possui um assento e um encosto. Nesse momento, organiza-se no pensamento o conceito dessa palavra, focando a classe dos móveis e excluindo outras classes (CAFÉ; SALES, 2010).

No contexto dos sistemas de informação não é diferente. Os sistemas organizam um acervo, cuja função é armazenar objetos informacionais, compreendendo melhor de que tratam, dispondo de forma ordenada, de maneira que as informações neles registradas possam ser recuperadas com facilidade. Essa prática de armazenar e organizar o conteúdo informacional produzido, visando alcançar usuários futuros, não é recente, como salienta McGarry (1999, p. 112): “[...] o anseio de armazenar os produtos da memória coletiva tem estado conosco desde o início da era da escrita."

Antes de prosseguir com a abordagem acerca do conceito de Organização da Informação, seu significado e práticas, é importante diferenciá-lo do conceito de Organização do Conhecimento. Assim, Brascher e Café (2008), que discorrem em seu trabalho sobre a divergência entre os conceitos, abordam a existência de uma discordância entre a delimitação de Organização da Informação (OI) e Organização do Conhecimento $(\mathrm{OC})$ que, por vezes, são utilizados com falta de clareza em seus sentidos.

Para as autoras, anteriormente citadas, a Ol se aplica às ocorrências individuais de objetos informacionais, compreendendo a organização desses objetos para arranja-los sistematicamente em coleções. A OC, por sua vez, se aplica às unidades do pensamento (conceitos), e visa a construção de modelos de mundo que 
se constituem em abstrações da realidade, que permitem descrever e fornecer explicações sobre os fenômenos que podem ser observados.

De forma resumida, a Ol tem como objeto a informação registrada passível de ser armazenada e organizada, possibilitando sua recuperação quando necessário. Já a $O C$ se encontra no mundo dos conceitos que visam à construção de representações do conhecimento. A figura 1, a seguir, ilustra essa proposta em relação a diferenciação entre os dois conceitos

Figura $1-\mathrm{OC} / \mathrm{RC}$ e Ol/RI

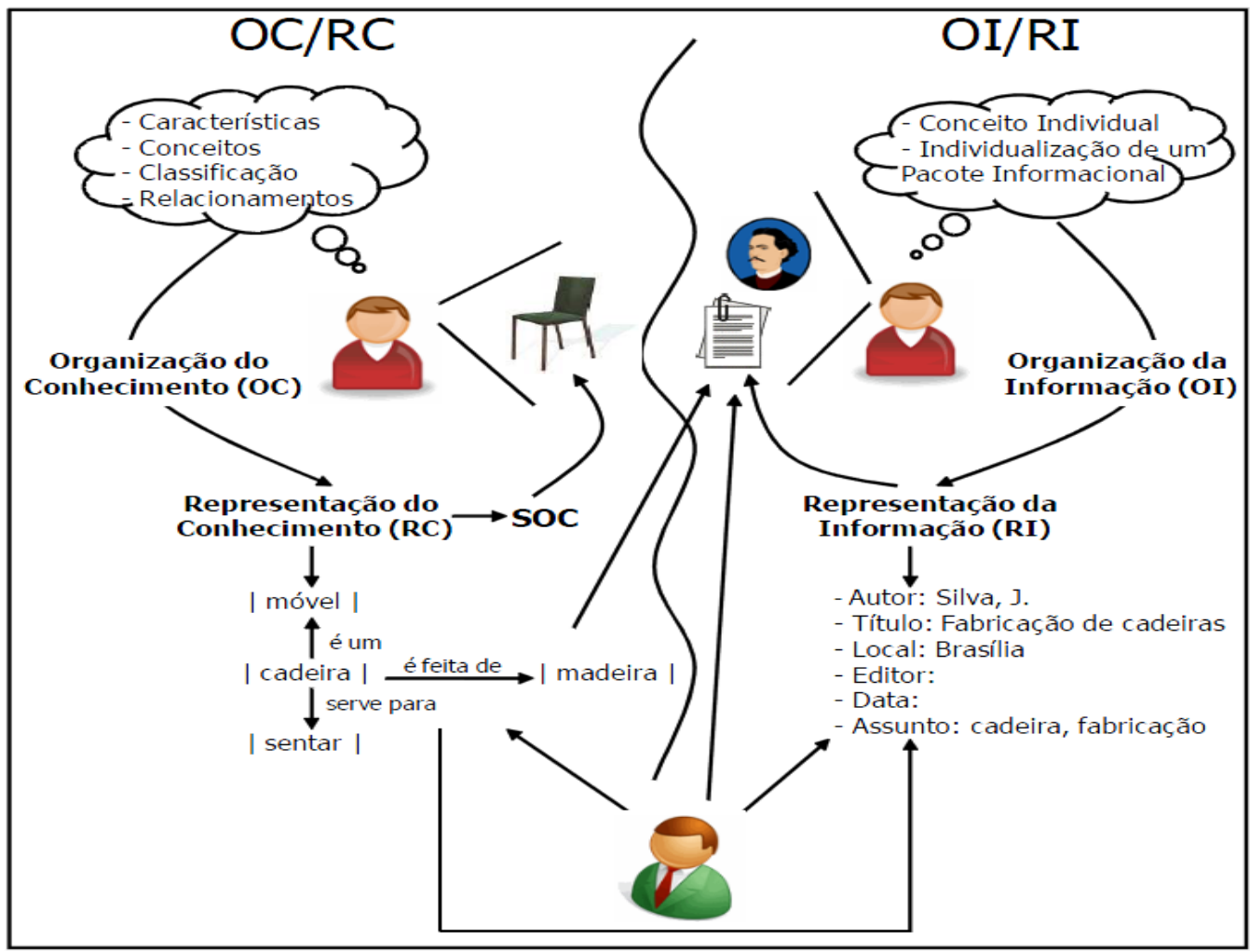

Fonte: Brascher e Café (2008, p. 7).

Com base no exposto acerca da OI e da OC, optou-se aqui pela utilização do termo Organização da Informação, já que sua proposta se adequa ao objetivo que se pretende alcançar com esta pesquisa, ou seja, analisar os aspectos da Ol e sua influência na prática da organização que resulta na facilitação do acesso e recuperação de documentos informacionais em SRIs.

Dessa forma, considera-se a proposta conceitual de Brascher e Café (2008), em que a Ol compreende a organização de um conjunto de objetos informacionais para arranjá-los sistematicamente em coleções, como a organização da informação em bibliotecas, museus, arquivos, tanto tradicionais quanto eletrônicos. Considera- 
se, também, a contribuição de Alvarenga (2003), que acrescenta ainda que os processos de organização da informação destinam-se, prioritariamente, à recuperação eficaz de informações por parte dos usuários.

Assim, para melhor compreender como se desenvolvem as práticas da $\mathrm{OI}$, se faz necessário uma análise mais aprofundada a respeito de um aspecto essencial que fundamenta suas atividades - o tratamento da informação.

\subsection{Tratamento da Informação}

Contextualizando o tratamento da informação em sistemas de informação, Dias $(2001$, p. 3) o define como "a função de descrever os documentos, tanto do ponto de vista físico (características físicas dos documentos) quanto do ponto de vista temático (ou de descrição do conteúdo)." Sendo assim, o tratamento da informação busca representar os documentos, através da síntese deles, para que sua organização e manipulação dentro do sistema de recuperação possam ser facilitadas e para que o usuário possa avaliar, de maneira mais simples, a relevância que os documentos apresentam para sua necessidade de informação.

Nessa linha de pensamento, Foskett (1973 apud GUIMARÃES, 2009) reconhece que a Ol é uma atividade de natureza eminentemente mediadora, em que dois universos se descortinam: o primeiro, ligado ao acesso físico aos documentos; e o segundo, de natureza mais complexa, voltado para o acesso ao conteúdo informacional. Desse modo, a organização da informação é composta por dois grandes processos que estão relacionados entre si: o tratamento descritivo e o tratamento temático.

Após análise da OI e dos processos que fundamentam as suas atividades, se faz relevante abordar a VI, enquanto área que contribui, por meio da construção de interfaces gráficas, para representação visual de conteúdo informacional e atua no intuito de facilitar o acesso às informações que foram previamente organizadas.

\section{VISUALIZAÇÃO DA INFORMAÇÃO}

Nesse sentido, a VI pode ser definida como uma área da ciência que tem por objetivo o estudo das principais formas de representações gráficas para apresentação de informações, a fim de contribuir para o melhor entendimento delas, 
bem como ajudar a percepção do usuário na dedução de novos conhecimentos baseado no que está sendo apresentado (FREITAS et al., 2001).

Outra interessante definição é trazida pelo grupo de pesquisa em VI do instituto de pesquisa de software da Universidade da Califórnia, Irvine ${ }^{1}$, segundo o qual a VI está centrada no desenvolvimento e análise de métodos para a apresentação de informações abstratas de forma visual. Tendo em vista que a representação visual de informações permite que as pessoas se tornem mais facilmente consciente de fatos essenciais, desenvolvendo uma compreensão mais profunda sobre o que está sendo apresentado e permitindo, através de uma visualização interativa, manipular e explorar informações.

Resumidamente, a VI está fundamentada no fato de que a informação apresentada de maneira visual possibilita uma compreensão mais clara daquilo que está sendo transmitido, já que, como acrescenta Dias e Carvalho (2007, p.1):

[...] na maioria dos casos o oferecimento de imagens, figuras, estruturas gráficas e quaisquer outros recursos gráficos, com a finalidade de apresentar uma informação, produz a compreensão da mensagem transmitida, pois esta se torna mais natural e exige menos esforço cognitivo.

Esse ponto de vista também é compartilhado por Zhang (2008) ao relatar que os seres humanos são atraídos por imagens e informação visual. Segundo Zhang, as imagens e gráficos podem ser cativantes e atraentes, especialmente, se bem elaborados, pois uma representação visual pode comunicar informações de maneira mais rápida e eficaz que qualquer outro método. Como exemplo básico, pode-se considerar a diferença entre uma descrição escrita do rosto de uma pessoa e uma fotografia dela, nesse caso a informação transmitida pela imagem poderá gerar uma compreensão mais clara e precisa acerca da pessoa à quem se refere do que a informação descritiva.

Estivalet (2000) também reafirma essa idéia ao ressaltar que se torna fácil para uma pessoa capturar o significado de uma imagem ao invés de vários dados isolados, pois uma representação visual proporciona uma forma simples e intuitiva de entender o significado das informações a serem transmitidas.

\footnotetext{
${ }^{1}$ Informações em: http://www.isr.uci.edu/research.html
} 
Nesse aspecto, é importante destacar que, para que seja possível a construção dessas representações visuais, a VI trabalha em parceria com as ferramentas computacionais, que se configuram como facilitadoras dos processos de visualização. A esse respeito, Mazza (2009) explica que, embora a visualização não dependa necessariamente de computadores para cumprir seu papel, por ser um processo que acontece na mente, as ferramentas computacionais advindas do avanço da tecnologia têm o poder de potencializar o uso de visualizações, através de recursos modernos e interativos.

Ware (2004) e Card et al. (1999 apud PRESSER; SILVA; SANTOS, 2010) pontuam, nesse sentido, que a VI procura reunir ambos, o poder da percepção visual humana e o poder de processamento do computador, para analisar e compreender um conjunto de informações. Assim, com base nessa colocação, podese entender que a VI, tendo como objetivo final a inclusão informacional dos usuários, conta, de maneira geral, com o auxílio da tecnologia e dos computadores para geração de representações gráficas, se apoiando no uso da capacidade visual humana que, como afirma Nascimento e Ferreira (2005), é o sentido que possui maior capacidade de captação de informações por unidade de tempo.

Como visto até o momento, a VI, enquanto atividade temática, está voltada para as questões que envolvem a otimização na representação e, consequentemente, no acesso, localização e recuperação de informações.

Buscando compreender de forma mais clara a influência que a visualização exerce na recuperação de informação, se faz relevante destacar a visão de Zhang (2008) ao elucidar que o dinamismo, a diversidade, a heterogeneidade e a complexidade de informações dispostas na internet e em outros sistemas de recuperação de informação têm representado um desafio para as técnicas e teorias tradicionais de recuperação de informação. Esses desafios levam a necessidade de adoção de uma maneira mais interativa, intuitiva e eficaz de recuperação de informação nos sistemas. Nessa perspectiva, encontra-se a VI que, por meio das interfaces gráficas, se configura como uma poderosa aliada em SRIs.

Assim, quando tais sistemas possuem suas informações devidamente organizadas, a utilização de interfaces gráficas pode vir a ser um potencializador na representação e, consequentemente, na recuperação, minimizando algumas das dificuldades enfrentadas pelos sistemas, já que o uso dessas interfaces facilita a 
interação com o usuário e permite, como afirma Mazza (2009), entender sistemas complexos, tomar decisões e descobrir informações importantes que, de outra maneira, poderiam permanecer ocultas.

Além do mais, a adoção de interfaces gráficas por SRls tem se tornado cada vez mais acessível, pois o avanço das tecnologias de informação, dos equipamentos de imagens em geral e dos computadores tem tornado possível a construção de sistemas complexos e ricos na qualidade da apresentação de suas informações, através de recursos gráficos e de interfaces cada vez mais interativas e de fácil utilização.

\section{METODOLOGIA}

Nesta seção apresenta-se a metodologia que caracteriza a pesquisa, bem como o percurso metodológico utilizado para sua realização.

\subsection{Tipo de Pesquisa}

Esta pesquisa se caracteriza como exploratória, uma vez que, para o alcance dos objetivos propostos, buscou-se levantar e explorar referencial teórico acerca da organização e representação visual de informações como alternativa para potencializar o acesso e a recuperação do conteúdo informacional armazenado em SRIs. Assim, por meio da pesquisa exploratória, que, de acordo com Gil (2009, p. 27) "[...] tem como principal finalidade desenvolver, esclarecer [...] conceitos e ideias, tendo em vista a formulação de problemas mais precisos [...]", buscou-se proporcionar uma maior familiaridade com as áreas abordadas.

Com o intuito de possibilitar a formação de um referencial teórico acerca das temáticas aqui estudadas, a pesquisa se caracteriza, também, como bibliográfica. Pois, a partir da pesquisa bibliográfica obtém-se o embasamento científico para melhor compreensão e análise de cada área.

\subsection{Levantamento Bibliográfico}

Inicialmente, foi feito um levantamento bibliográfico baseado em literatura voltada para as temáticas aqui abordadas, com o intuito de fundamentar a pesquisa, e, para isso, foram analisadas referências teóricas extraídas de artigos, teses, 
dissertações e livros, presentes na literatura nacional e internacional, que versem sobre Organização da Informação, Visualização da Informação e Sistemas de Recuperação de Informação. As buscas por artigos foram realizadas, também, nas Bases de Dados: BRAPCI e SCIELO; e no portal de periódicos da CAPES.

\subsection{Descrição de Técnicas e Modelos de Interface de VI}

Em seguida, buscou-se abordar as técnicas e modelos de interface de visualização da informação, sendo analisados aqueles que melhor se adéquam aos SRIs, ou seja, que tenham aplicações práticas na construção de interfaces para sistemas que lidam com a recuperação da informação, possibilitando a representação e visualização da estrutura da coleção de documentos.

Nessa perspectiva, quanto às técnicas, foram analisadas: - Desenho de Grafos; - Desenho de Árvores; - Browser Hiperbólico.

Quanto aos modelos de VI foram analisados os seguintes: - Mapa Conceitual; - Cartografia Temática; - Nuvem de Tags.

\subsection{Análise de Sites de SRls}

Por último, foi feito um levantamento de SRIs na Web que adotam visualizações na interface com o usuário. Nesse contexto, apresenta-se uma análise de como deveriam estar organizadas e estruturadas as informações e quais instrumentos seriam necessários para tornar possível a aplicação eficaz de cada técnica e modelo de VI nos sistemas. Para a análise, foram abordados os seguintes sites de SRIs e suas interfaces visuais:

- AquaBrowser da biblioteca da Universidade de Edimburgo - o catálogo on line da biblioteca faz uso de interface baseada na técnica desenho de grafos;

- Mecanismo de busca YIPPY - faz uso do desenho de árvores de diretórios para organizar os documentos retornados para uma consulta realizada em sua base de documentos;

- Infoteca da Embrapa - faz uso da técnica Browser Hiperbólico para permitir acesso a informações sobre tecnologias produzidas pela Embrapa;

- Website do IHMC Camp Tools - utiliza o mapa conceitual como modelo de interface para descrever o programa Cmap Tools; 
- WEBSOM - método para construção de mapas visuais - utiliza a cartografia temática como um método para gerar mapas visuais de assuntos;

- Sistema de busca da BRAPCI - faz uso da nuvem de tags como interface visual para auxiliar o usuário, sugerindo termos de busca.

\section{RESULTADOS}

Nesta seção será apresentado o resultado alcançado a partir da análise da relação entre OI e VI em sites de SRIs.

\subsection{Sistemas que Fazem uso da Visualização da Informação}

Busca-se, a seguir, abordar alguns sites de SRIs que utilizam interface visuais em sua estrutura, demonstrando, de forma prática, como a aplicação de visualizações pode auxiliar na localização e recuperação de informação.

Nesse contexto os sistemas são analisados quanto as técnicas e modelos de interface de VI adotados em sua estrutura, levando em conta que, por meio de suas técnicas e modelos de interface, a VI pode ser considerada uma poderosa aliada para os sistemas que se propõem a oferecer uma interface amigável e interativa para acesso, localização e recuperação das informações por eles armazenadas. Pois, como afirmam Torres Ponjuan e Ponjuan Dante (2010), o uso de recursos visuais para representação de informação possibilita o rápido acesso, localização e compreensão do que está sendo transmitido. Maiores detalhes acerca das técnicas e modelos de VI podem ser encontrados em (CORREA; VIEIRA; 2013).

Por outro lado, os sistemas são analisados sob a perspectiva da organização da informação, ou seja, buscou-se compreender como deveriam estar organizadas as informações nas bases de dados dos SRls e quais instrumentos seriam necessários para tornar possível a construção de uma interface visual para esses sistemas, tendo em vista que a organização prévia das informações, bem como a adoção de instrumentos adequados para isso, são elementos necessários para a representação de informações por meio de estruturas de VI.

Nesse contexto, buscando demonstrar, de maneira resumida, os resultados obtidos por meio da análise realizada nos sites de SRI quanto à interação entre OI e 
$\mathrm{VI}$, apresenta-se, a seguir, um quadro que permite visualizar as técnicas e modelos de interface visual adotados por cada um dos sites, bem como os instrumentos de Ol adequados para a construção e funcionamento de visualizações expressivas.

Quadro 1 - Relação entre OI e VI na aplicação de interface visual

\begin{tabular}{|c|c|c|c|}
\hline Site de SRI & $\begin{array}{l}\text { Técnica ou } \\
\text { modelo de } \\
\text { VI }\end{array}$ & Interface Visual & $\begin{array}{l}\text { Instrumentos de Ol para } \\
\text { o emprego de } \\
\text { visualização }\end{array}$ \\
\hline $\begin{array}{l}\text { 1- } \\
\text { AquaBrowser } \\
\text { da biblioteca } \\
\text { da } \\
\text { Universidade } \\
\text { de Edimburgo }\end{array}$ & $\begin{array}{l}\text { Desenho de } \\
\text { Grafos }\end{array}$ & 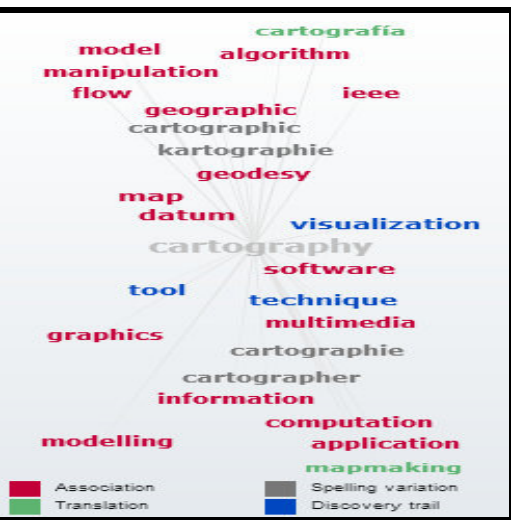 & $\begin{array}{l}\text { Adoção de um tesauro ou } \\
\text { taxonomia como } \\
\text { instrumento de Ol que } \\
\text { permita uma representação } \\
\text { e organização adequada } \\
\text { de termos e que } \\
\text { estabeleçam as relações } \\
\text { existentes entre conceitos. }\end{array}$ \\
\hline $\begin{array}{l}\text { 2- Mecanismo } \\
\text { de busca } \\
\text { YIPPY }\end{array}$ & $\begin{array}{l}\text { Desenho de } \\
\text { Árvores }\end{array}$ & 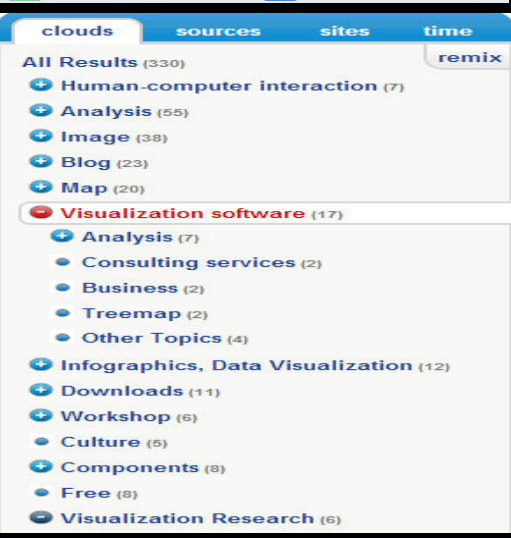 & $\begin{array}{l}\text { Adoção de uma } \\
\text { taxonomia como } \\
\text { instrumento de Ol que } \\
\text { permita a categorização } \\
\text { de conteúdos } \\
\text { semelhantes, } \\
\text { evidenciando as relações } \\
\text { hierárquicas existentes } \\
\text { entre eles. }\end{array}$ \\
\hline $\begin{array}{l}\text { 3- Infoteca-e } \\
\text { da Embrapa }\end{array}$ & $\begin{array}{l}\text { Browser } \\
\text { Hiperbólico }\end{array}$ & 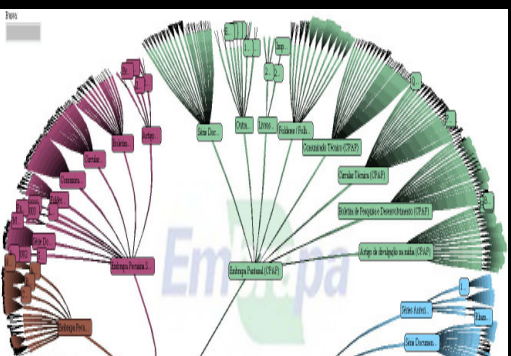 & $\begin{array}{l}\text { Adoção de uma taxonomia } \\
\text { como instrumento capaz de } \\
\text { organizar termos em } \\
\text { categorias da mais } \\
\text { genérica a mais específica, } \\
\text { estabelecendo relações } \\
\text { hierárquicas entre eles. }\end{array}$ \\
\hline
\end{tabular}



recuperação de informação

\begin{tabular}{|c|c|c|c|}
\hline $\begin{array}{l}\text { 4- Website do } \\
\text { IHMC } \\
\text { CampTools }\end{array}$ & $\begin{array}{l}\text { Mapa } \\
\text { Conceitual }\end{array}$ & 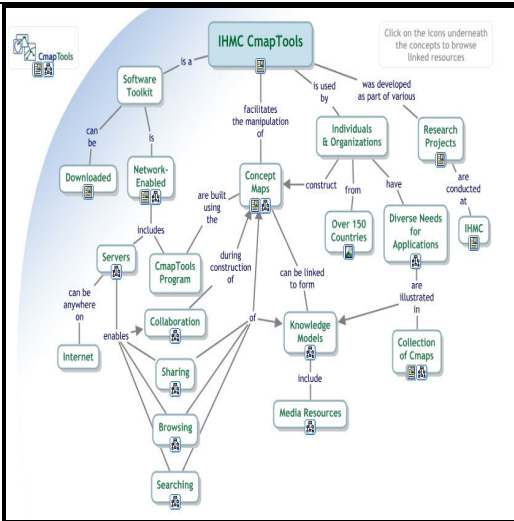 & $\begin{array}{l}\text { Adoção de ontologia como } \\
\text { instrumento que possibilite } \\
\text { congregar conjuntos de } \\
\text { conceitos padronizados, } \\
\text { termos e definições, } \\
\text { evidenciando a forma com } \\
\text { que esses conceitos estão } \\
\text { relacionados entre si. }\end{array}$ \\
\hline $\begin{array}{l}\text { 5- WEBSOM - } \\
\text { método para } \\
\text { construção de } \\
\text { mapas visuais. }\end{array}$ & $\begin{array}{l}\text { Cartografia } \\
\text { Temática }\end{array}$ & 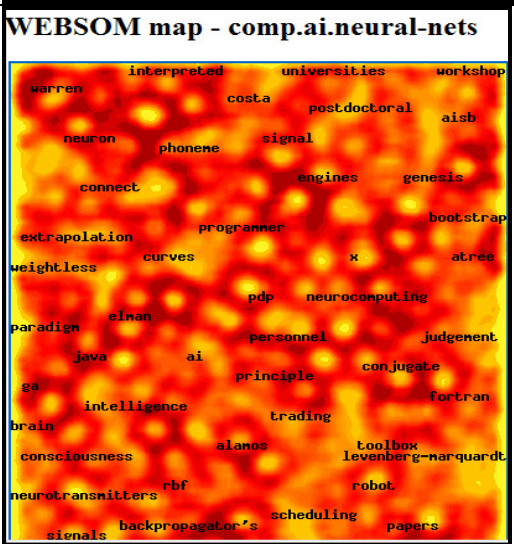 & $\begin{array}{l}\text { Adoção da ontologia } \\
\text { enquanto instrumento que } \\
\text { permite a representação e } \\
\text { a categorização de } \\
\text { documentos, bem como o } \\
\text { estabelecimento de } \\
\text { temáticas relacionadas; } \\
\text { adoção de critérios para } \\
\text { identificar a coocorrência } \\
\text { entre termos. }\end{array}$ \\
\hline $\begin{array}{l}\text { 6- Sistema de } \\
\text { busca da } \\
\text { BRAPCI }\end{array}$ & $\begin{array}{l}\text { Nuvem de } \\
\text { Tags }\end{array}$ & 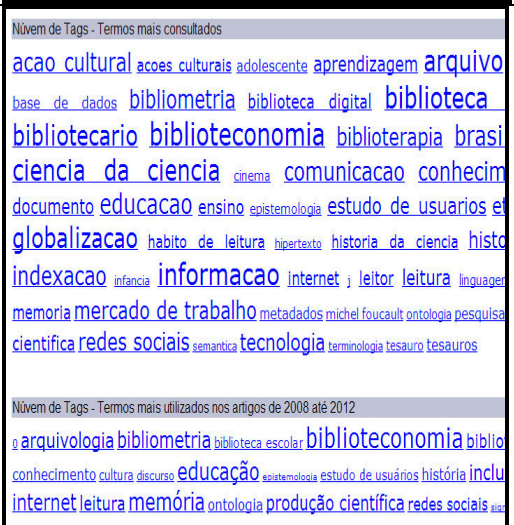 & $\begin{array}{l}\text { Adoção de listas de } \\
\text { cabeçalhos de assunto } \\
\text { para auxiliar na } \\
\text { organização, estruturação } \\
\text { e representação das tags } \\
\text { que servirão como pontos } \\
\text { de acesso para localização } \\
\text { de documentos. }\end{array}$ \\
\hline
\end{tabular}

Fonte: Elaborado pela autora

Assim, por meio do quadro 1, é possível visualizar os sites analisados, as técnicas e modelos de $\mathrm{VI}$ adotados por cada um, a figura ilustrando a interface visual, bem como os instrumentos de Ol adequados para permitir uma eficaz organização e recuperação de informações. De maneira mais detalhada, tem-se:

\section{Site 1- AquaBrowser da biblioteca da Universidade de Edimburgo, que} faz uso da técnica de VI "Desenho de Grafos" para disponibilizar o catálogo da biblioteca por meio de interface gráfica. A técnica Desenho de Grafos permite que, a partir de uma busca expressa na caixa de texto, o sistema gere uma visualização 
apresentando os resultados para essa busca por meio de termos, representados por vértices, e das relações entre eles por arestas. Dessa forma, o termo utilizado na busca permanece no centro da figura enquanto os termos relacionados a ele estão ligados por meio das arestas, sendo o tipo de relação estabelecida entre os termos determinada pela cor dos vértices, indicando associações, traduções, variações de ortografia e a trilha (rota) de pesquisa.

Quanto aos instrumentos de Ol que podem ser adequados para organizar as informações no sistema de modo a evidenciar as relações existentes entre conceitos, tem-se o tesauro e a taxonomia. Os tesauros são capazes de organizar termos relacionados semântica e genericamente, e são considerados, conforme Carlan e Medeiros (2011), instrumentos de controle terminológico eficazes para a organização de conceitos e importantes ferramentas no tratamento e recuperação da informação.

No mesmo contexto está inserida a taxonomia, como instrumento que permite agrupamento categorizado de assuntos e a construção de relacionamentos semânticos entre termos, sendo considerada, também, como menciona Vital (2012, p. 26), "uma metodologia facilitadora tanto da organização quanto da recuperação, representação e disponibilização do conteúdo em ambiente Web."

Site 2- Mecanismo de busca YIPPY, trata-se de um sistema de busca que faz uso de interface visual baseada na técnica "Desenho de Árvores" para organizar os itens recuperados, agregados em categorias e dispostos em uma lista ordenada, por meio de uma interface simples e intuitiva. A técnica Desenho de Árvores permite dispor conteúdos por hierarquias, por meio de uma árvore de diretórios, podendo disponibilizar itens por tópicos e subtópicos de acordo com a semelhança entre eles.

Nesse contexto, se faz necessário a análise de um instrumento de Ol que auxilie na organização de conceitos, de maneira a possibilitar que a visualização cumpra o seu objetivo, ou seja, disponibilizar, de maneira organizada e simplificada, os resultados de uma busca. Sendo assim, pode-se citar a taxonomia como instrumento que busca estabelecer categorias para a informação e apresentam relacionamentos semânticos entre termos (VITAL, 2012). Assim, se torna possível apresentar os resultados de uma pesquisa por meio da categorização ou agrupamento de conteúdos semelhantes.

Site 3 - Infoteca-e da Embrapa, para fornecer acesso às informações sobre 
tecnologias produzidas pela Embrapa (Empresa Brasileira de Pesquisa Agropecuária) esse site adota uma árvore hiperbólica, isto é, uma interface visual em forma de árvore espacial, baseada na técnica browser hiperbólico. Essa interface visual permite que os conceitos estejam organizados por hierarquias (categorias) tendo um assunto mais geral centralizado e, dentro deste, assuntos específicos relacionados a ele, por meio de vértices em uma espécie de árvore.

$\mathrm{Na}$ estrutura, dentro de cada assunto específico estão dispostos os termos que se ligam a ele, por meio de ramificações que condensam os assuntos cada vez mais específicos, sendo possível, através desta estrutura, visualizar desde os assuntos mais abrangentes até aqueles menos inclusivos dentro da árvore, possibilitando a compactação das informações à medida que se aproxima da periferia do desenho.

A eficaz utilização dessa técnica remete à necessidade de um instrumento de Ol que permita uma organização baseada no estabelecimento de relações hierárquicas entre conceitos. Nesse contexto, se insere a taxonomia como instrumento voltado à organização de informações em ambientes específicos e que, como afirma Vital (2012), é caracterizado pela sua estrutura hierárquica, capaz de organizar termos em categorias da mais genérica a mais específica, estabelecendo relações entre conceitos.

Site 4 - Website do IHMC CampTools, apresenta um software, desenvolvido pelo IHMC (Institute for Human \& Machine Cognition), que permite construir, navegar, compartilhar e criar modelos de conhecimento representados como mapas conceituais. Esse website disponibiliza um mapa para descrever a ferramenta por eles desenvolvida e para ilustrar o funcionamento de "Mapa Conceitual", enquanto modelo de interface visual para organização e disponibilização de informações. Esse modelo de interface é formado por conceitos, geralmente localizados em círculos ou retângulos de algum tamanho, e por relacionamentos entre esses conceitos, que são indicados por uma linha que os conecta, formando proposições. Palavras sobre as linhas especificam as relações entre esses conceitos.

Para organizar as informações a serem dispostas por meio do mapa conceitual, pode-se citar como alternativa de instrumento de Ol as ontologias, já que são conhecidas pela potencialidade em organizar e representar informação. As ontologias, como menciona Silva (2008), são estruturas que congregam conjuntos 
de conceitos padronizados, termos e definições, evidenciando a forma com que esses conceitos estão relacionados entre si. Nessa linha de pensamento, Brandão e Lucena (2002) enfatizam que o uso de ontologias possibilita não só a definição de conceitos num domínio de conhecimento, como também permite a definição de relacionamentos entre esses conceitos e de inferências básicas os envolvendo.

Site 5 - WEBSOM - método para construção de mapas visuais, é um método para construção de mapas visuais que organiza coleções de documentos e permite a exploração e recuperação desses documentos por meio de uma interface visual baseada na técnica "Cartografia Temática". Essa técnica permite que os documentos armazenados estejam representados e organizados por meio de termos, esses termos indicam o contexto dos documentos e ficam agrupados em pontos do mapa de acordo com a similaridade entre os conteúdos que representam, ou seja, a proximidade entre os termos indica que eles abordam temáticas relacionadas. Essa relação de associação entre os documentos é medida pela coocorrência entre termos. Dessa forma, os documentos semelhantes estão posicionados em grupos próximos uns dos outros no mapa.

Para que a técnica possa ser aplicada se faz necessário a adoção de um instrumento que permita representar os documentos por meio de um vocabulário controlado, que seja capaz de expressar as relações associativas entre os documentos. Sob essa perspectiva, pode-se citar como instrumento adequado ao contexto da cartografia temática as ontologias, tendo em vista que, como já mencionado por Silva (2008), as ontologias são estruturas que congregam conjuntos de conceitos padronizados, termos e definições aceitas por uma comunidade particular e a forma como esses conceitos estão relacionados entre si.

Site 6 - Sistema de busca da BRAPCI (Base de Dados Referenciais de Artigos de Periódicos em Ciência da Informação) adota visualizações em forma de "Nuvem de Tags" como uma alternativa amigável para a busca e identificação de conteúdos. Essa interface é composta por palavras-chave dispostas em uma lista visual com o objetivo de representar itens de conteúdo de um sistema. A nuvem de tags é, também, onde se concentram as tags mais utilizadas e/ou vistas no momento, podendo funcionar como pistas para os usuários, pois tem a função de facilitar a navegação, dando orientações e indicando por onde esses usuários poderiam caminhar para localização de informações de seu interesse. 
Quanto ao instrumento a ser adotado para que esse tipo de interface possa ser utilizado, têm-se os cabeçalhos de assunto, tendo em vista que são instrumentos que uniformizam a descrição sintética dos assuntos contidos nos documentos, servindo como pontos de acesso para sua localização. Os cabeçalhos de assunto são elaborados a partir de palavras simples ou compostas (ordenadas em lista alfabética), geralmente extraídas do próprio documento, para descrever seus assuntos. Nas listas formadas pelos cabeçalhos de assunto podem ser encontradas, ainda, remissivas de orientação para assuntos relacionados como, por exemplo: "linguagem documental", ver também "classificação", "documentação", "linguística" (GUINCHAT; MENOU, 1994).

As listas de cabeçalhos de assunto podem ser, portanto, úteis à organização da informação na nuvem de tags, visto que podem auxiliar na estruturação, representação e padronização das tags, bem como possibilitar a identificação e recuperação de documentos sobre uma determinada temática ou sobre temáticas relacionadas.

\subsection{Relação entre OI e VI nos sites de SRIs que Fazem uso de Interface Visual}

Com base nas análises realizadas anteriormente acerca dos SRIs que adotam interfaces visuais em sua estrutura, é possível argumentar que, embora os sites de SRIs analisados apresentem propostas e funcionalidades diversificadas, todos eles caminham para os mesmos objetivos - facilitar a visualização, navegação, descoberta e recuperação de informações. Para alcançar esses objetivos, os sites adotam técnicas e modelos de interface visual que melhor se adéquam aos seus contextos e particularidades. Em decorrência da adoção das interfaces visuais, esses sites oferecem diversos benefícios aos usuários, como:

- Oferecem uma maneira amigável e dinâmica de interação com as informações dispostas;

- Auxiliam na compreensão de como estão estruturadas as informações;

- Facilitam na localização de informações precisas;

- Contribuem para descoberta e dedução de novas informações,

- Permitem a visualização de conteúdos diferentes que antes eram desconhecidos, mas que podem vir a ser pertinentes à necessidade do 
usuário;

- Minimizam a sobrecarga de informação sobre o usuário.

Outro ponto importante a ser analisado quando se refere à aplicação de interfaces de $\mathrm{VI}$ em SRIs diz respeito aos aspectos que envolvem a organização prévia das informações a serem representadas.

A esse respeito, identificou-se a influência que a Ol exerce, por meio de seus instrumentos, para auxiliar na organização e representação de conteúdos que serão transmitidos em estruturas visuais, confirmando, dessa forma, que sistemas que fazem uso de visualizações para transmitir informação requerem métodos de OI adequados para dar suporte às representações visuais de informação e para permitir a recuperação de conteúdos, já que, como afirma Zhang (2008), os métodos de organização e armazenamento de informações afetam e determinam os métodos de recuperação de informação.

Sob essa perspectiva, é importante elucidar que a atuação desses instrumentos de Ol é direcionada para a organização das informações a serem transmitidas e que essa organização poderá ser refletida de maneira visual, podendo acarretar em visualizações expressivas, capazes de permitir representação facilitada, por meio de estrutura simples e recuperação satisfatória de informações.

\section{CONSIDERAÇÕES FINAIS}

Os SRIs, enquanto sistemas que lidam com informação registrada e que trabalham com o propósito de permitir, a partir de um universo de documentos disponíveis, a recuperação daqueles que venham a ser úteis para satisfação de uma necessidade informacional, exercem um importante papel em uma sociedade caracterizada pela crescente produção e disseminação de informação, amplamente influenciada pelas tecnologias de informação e comunicação disponíveis.

Nesse contexto, os SRIs podem ser vistos como sistemas de armazenamento, preservação e acesso à memória humana produzida, registrada e por eles armazenada, o que confere a eles, como visto anteriormente, o título de sistemas detentores de memória. Esse título atribui a eles uma importante missão, a de tornar acessível uma quantidade cada vez maior de conteúdo informacional, o 
que implica numa necessidade por meios precisos de acesso à informação elemento vital às atividades humanas - bem como de maior controle sobre essa informação, com vista a prover a sua socialização e recuperação.

Considerando essas necessidades, partiu-se do pressuposto que a Ol e a VI, enquanto áreas que assumem uma relação direta com a recuperação da informação, podem ser consideradas poderosas aliadas para os SRls. Neste sentido, buscou-se uma análise mais aprofundada acerca dessas áreas, o que resultou em uma compreensão mais clara sobre as particularidades de cada uma e tornou possível identificar a potencial ligação entre elas. A figura 2 permite, por meio de uma visualização sucinta, ilustrar essa questão.

Figura 2 - Análise do foco das áreas de OI e VI

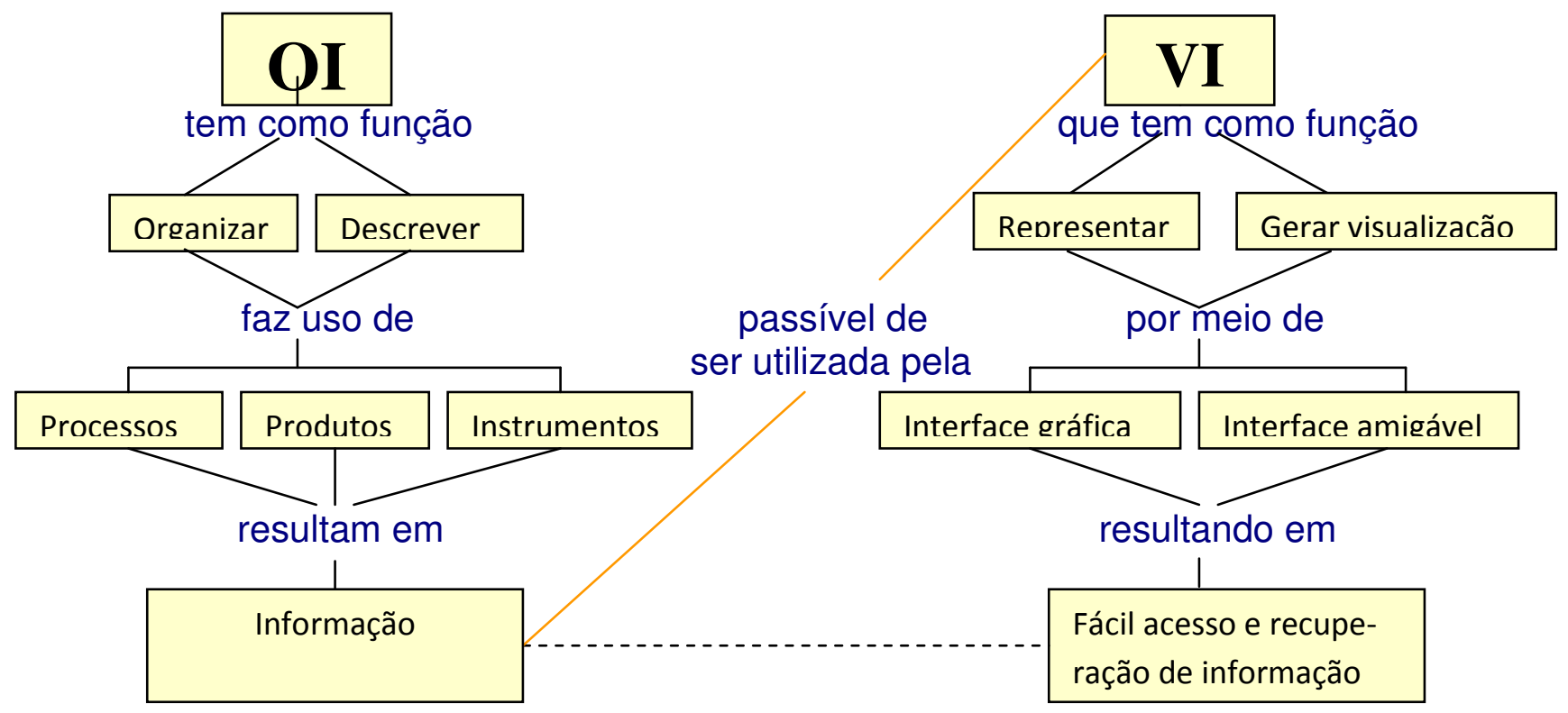

Fonte: Elaborado pela autora.

A partir do que foi abordado na revisão de literatura acerca da OI e VI, foi possível evidenciar (conforme demonstrado na figura 2) que a OI, enquanto área que se preocupa com a organização, descrição e tratamento da informação, fazendo uso, para isso, de processos, produtos e instrumentos, pode subsidiar a construção de interfaces de $\mathrm{VI}$, cujo objetivo é representar informações de forma gráfica, facilitando o acesso e recuperação de conteúdos informacionais, por meio de uma interface amigável. 
Dessa forma, entende-se que a Ol é capaz de oferecer um suporte para que a $\mathrm{VI}$ exerça sua função de forma satisfatória, pois para que a VI potencialize o acesso e a recuperação da informação, o ideal é que essa informação esteja prévia e adequadamente organizada. Entende-se, assim, que a Ol e a VI possuem focos específicos, mas que podem caminhar em harmonia para um objetivo comum: possibilitar o acesso e recuperação eficaz de informação organizada.

Foi exatamente nesse contexto que se desenvolveu o objetivo proposto por esta pesquisa, ou seja, buscou-se analisar a Ol e a VI sob o ponto de vista da contribuição dessas áreas para os SRIs. Para isso, como validação do que foi pesquisado durante a revisão de literatura, foram analisados sites de SRIs que fazem uso de interfaces de $\mathrm{VI}$, com o intuito de evidenciar as vantagens oferecidas pelo uso de visualizações nesses sistemas, bem como permitir a análise dos requisitos de Ol necessários para tornar possível a construção das interfaces visuais e a recuperação de conteúdos informacionais relevantes.

Por meio da análise desses sites pode-se comprovar que, de fato, tem-se nas interfaces de VI ricas alternativas para representação de conteúdo informacional, disponibilizando uma maneira interativa para visualização, localização e recuperação das informações armazenadas. Além disso, sob a perspectiva da OI, foi possível compreender a influência que a adoção de seus instrumentos exercem na organização e representação de conteúdos que serão transmitidos em estruturas visuais. Verificou-se que a adoção de instrumentos adequados pode resultar em uma recuperação eficaz de informações, corroborando, assim, que os recursos de Ol afetam diretamente a recuperação da informação, conforme foi ressaltado ao longo da pesquisa.

Neste momento, é importante destacar que, embora não se possa afirmar que os sites analisados adotam em sua estrutura os instrumentos verificados como adequados nesta pesquisa, tais instrumentos foram sugeridos, pois entende-se que por meio da adoção deles se tem uma maior precisão na representação da informação, o que poderá possibilitar uma maneira expressiva de transmitir e recuperar informações por meio de interface visual, minimizando as chances de conteúdos relevantes permanecerem ocultos.

Em síntese, tem-se que os SRls, enquanto sistemas que detêm o importante papel de guardiões e propagadores da memória, no que se refere às informações 
registradas, necessitam de uma maior atenção à maneira como são abordados o acesso e a recuperação de informação. Essas necessidades estão alinhadas à questão básica da $\mathrm{Cl}$, que gira em torno do acesso à informação, ou facilitação desse acesso. Assim, fundamentando-se no fato de que a $\mathrm{Cl}$ está envolvida e empenhada nos aspectos que envolvem a recuperação da informação, bem como os meios de processá-la para otimizar sua acessibilidade e uso, considera-se, nesse cenário, a OI e a VI como potenciais auxiliadoras para os SRIs, considerando os preceitos de ambas as áreas e o papel que elas exercem quando se refere à informação, sua organização, visualização e recuperação.

Fundamentando-se na pesquisa desenvolvida, bem como nos autores referenciados neste trabalho chegou-se, por fim, à compreensão de que as referidas áreas, a saber, OI e VI, convergem entre si, ou seja, partilham de objetivos comuns e, a partir disso, defende-se que essas áreas podem alcançar promissores resultados, se atuarem em parceria para otimização na recuperação da memória armazenada em SRIs.

\section{REFERÊNCIAS}

ALVARENGA, Lídia. Representação do conhecimento na perspectiva da ciência da informação em tempo e espaço digitais. Encontros Bibli: Revista Eletrônica de Biblioteconomia e Ciência da Informação, Florianópolis, v. 8, n. 15, p. 18-40, 2003.

\section{ARAUJO JUNIOR, Rogério Henrique de. Precisão no processo de busca e recuperação da informação. Brasília: Thesaurus, 2007.}

ARAÚJO, Vania M. R. Hermes de. Sistemas de informação: nova abordagem teórico-conceitual. Ciência da Informação, Brasília, v. 24, n. 1, p. 1-39, jan./abr. 1995.

BRANDÃO, Anarosa Alves Franco; LUCENA, Carlos José Pereira de. Uma introdução à engenharia de ontologias no contexto da web semântica. Rio de Janeiro. Departamento de Informática, PUC-Rio, 2002. Disponível em:

$<\mathrm{ftp}: / / \mathrm{ftp}$.inf.puc-rio.br/pub/docs/techreports/02_29_brandao.pdf >. Acesso em: 10 out. 2013.

BRASCHER, Marisa; CAFÉ, Ligia. Organização da informação ou organização do conhecimento? In: ENCONTRO NACIONAL DE PESQUISA EM CIÊNCIA DA INFORMAÇÃO, 9., 2008, São Paulo. Anais... São Paulo: ENANCIB, 2008. p. 1-14.

BRASCHER, Marisa; CARLAN, E. Sistemas de organização do conhecimento: antigas e novas linguagens. In: ROBREDO, Jaime; BRÄSCHER, Marisa (Org.). 
Passeios pelo bosque da informação: estudos sobre a representação e organização da informação e do conhecimento - eroic. Brasília DF: IBICT, 2010. p. 148-176.

CAFÉ, Ligia; SALES, R. Organização da informação: conceitos básicos e breve fundamentação teórica. In: ROBREDO, Jaime; BRÄSCHER, Marisa. (Org.).

Passeios pelo bosque da informação: estudos sobre a representação e organização da informação e do conhecimento - eroic. Brasília DF: IBICT, 2010. p. 115-129.

CARLAN, Eliana; MEDEIROS, Marisa B. B. Sistemas de Organização do Conhecimento na visão da Ciência da Informação. RICl: revista lbero-americana de Ciência da Informação, Brasília,v. 4, n. 2, p. 53-73, ago./dez. 2011.

CORREA, Renato Fernandes; VIEIRA, Jessica Monique de Lira. Visualização da Informação aplicada à construção de interfaces para recuperação de informação na BDTD - UFPE. Perspectivas em Ciência da Informação, Belo Horizonte, v. 18, p. 18-34, out./dez. 2013.

DIAS, Eduardo Wense. Contexto digital e tratamento da informação.

DataGramaZero, Rio de Janeiro, v. 2, n. 5, p. 1-9, out. 2001.

DIAS, Mateus Pereira; CARVALHO, José Oscar Fontanini de. A visualização da informação e a sua contribuição para a ciência da informação. DataGramaZero, Rio de Janeiro, v. 8, n. 5, p. 1-16, out. 2007.

ESTIVALET, Luiz Fernando. 0 uso de ícones na visualização de informações. 2000. Dissertação (Mestrado em Ciência da Computação) - Universidade Federal do Rio Grande do Sul, Porto Alegre.

DAL' EVEDOVE, P. R. A política de tratamento da informação na percepção de catalogadores de assunto. In: CONGRESSO BRASILEIRO DE BIBLIOTECONOMIA, DOCUMENTAÇÃO E CIÊNCIA DA INFORMAÇÃO, 24., 2011, Maceió. Anais... Maceió: CBBD, 2011. p. 1-12.

FRAGOSO, Ilza da Silva. Instituições-memória: modelos institucionais de proteção ao patrimônio cultural e preservação da memória na cidade de João Pessoa-PB. 2008. 139f. Dissertação (Mestrado em Ciência da Informação) - Universidade Federal da Paraíba, João Pessoa.

FREITAS, Carla Maria Dal Sasso et al. Introdução à visualização de informações. RITA: Revista de Informática Teórica e Aplicada, Porto Alegre, v. 8, n. 2, p. 143-158, out. 2001.

FURTADO, Cassia. Educação e bibliotecas digitais. Revista digital de Biblioteconomia \& Ciência da Informação, Campinas, v. 8, n. 1, p. 103-116, jul./dez. 2010.

GIL, Antonio Carlos. Métodos e técnicas de pesquisa social. 6. ed. São Paulo: Atlas, 2009. 
GUIMARÃES, José Augusto Chaves. Abordagens teóricas de tratamento temático da informação (TTI): catalogação de assunto, indexação e análise documental. In: GARCÍA MARCO, F. J. Avances y perspectivas en sistemas de información y documentación. Ibersid, Zaragoza: Universidad de Zaragoza, 2009. p. 105-117.

GUINCHAT, Claire; MENOU, Michel. Introdução geral às ciências e técnicas da informação e documentação. Brasília: IBICT, 1994.

LE COADIC, Yves-Francois. A ciência da informação. 2. ed. Brasília: Briquet de Lemos/Livros, 2004.

LUNARDI, Márcia Severo. Visualização em nuvens de texto como apoio à busca exploratória na web. 2008. Dissertação (Mestrado em Design) - Escola Superior de Desenho Industrial, Universidade do Estado do Rio de Janeiro, Rio de Janeiro.

MAZZA, Riccardo. Introduction to information visualization. New York, NY: Springer, 2009.

MCGARRY, Kevin. O contexto dinâmico da informação: uma análise introdutória. Brasília: Briquet de Lemos/Livros, 1999.

NASCIMENTO, Hugo A. D.; FERREIRA, Cristiane. B. R. Visualização de informações: uma abordagem prática. In: CONGRESSO DA SOCIEDADE BRASILEIRA DE COMPUTAÇÃO, 25., 2005, São Leopoldo. Anais... São Leopoldo: UNISINOS, 2005. p. $1262-1312$.

PRESSER, Nadi Helena; SILVA, Eli Lopes da; SANTOS, Raimundo Nonato Macedo dos. Recursos de Formulação e Visualização de indicadores para apoiar processos de gestão educacional em IESS. Revista Digital de Biblioteconomia e Ciência da Informação, Campinas, v. 7, n. 2, p. 247-259, jan.jun. 2010.

SARACEVIC, Tefko. Interdisciplinary nature of information science. Ciência da Informação, Brasília, v. 24, n. 1, p. 36-41, jan./abr. 1995.

Ciência da informação: origem, evolução e relações. Perspectivas em Ciência da Informação, Belo Horizonte, v. 1, n. 1, p. 41-62, jan./jun. 1996.

SILVA, Daniela Lucas da. Uma proposta metodológica para construção de ontologias: uma perspectiva interdisciplinar entre as ciências da informação e da computação. 2008. Dissertação (Mestrado em Ciência da Informação) - Escola de Ciência da Informação, Universidade Federal de Minas Gerais, Belo Horizonte.

SVENONIUS, Elaine. The intellectual foundation of information organization. Cambridge: MIT Press, 2000.

TORRES PONJUAN, Deborah; PONJUÁN DANTE, Gloria. Influencias ergonómicas en la Visualización de Información. Informação \& Informação, Londrina, v. 15, n. 1, p. 17-36, jan./jun. 2010. 
VITAL, Luciane Paula. Taxonomia como elemento estruturante em portais corporativos. Revista Digital de Biblioteconomia e Ciência da Informação, Campinas, v. 9, n. 2, p. 25-40, 2012.

ZHANG, Jin. Visualization for information retrieval. New York: Springer, 2008.

Title

The contribution of Organization and Visualization Information for Information Retrieval Systems

Abstract

Introduction: Trying to propose strategies that enhance the understanding of memory stored in Information Retrieval Systems (IRSs), this thesis considers, as an alternative, the adoption of procedures of Information Organization (IO) and Information Visualization (IV).

Objective: Thus, this research aims to analyze and identify the relationship between the resources from the IO and IV from the point of view of the contribution of the two areas for SRIs, in order to enhance the understanding of memory stored by them, for that reason the research is characterized as exploratory and bibliographical.

Methodology: The methodological procedure used to conduct the research consists of bibliographic survey, for obtaining scientific basis for better understanding and analysis of each area; a survey of systems that make use of IV interface in its structure, as well as the analysis of the 10 criteria necessary to organization and representation of content to be transmitted in visual structures.

Results: From that, it was evident the interconnection between the areas addressed when applied in systems that deal with information retrieval.

Conclusions: Thus, it is concluded that the IO and IV can achieve promising results if they work in partnership to optimize the recovery of memory stored in IRSs.

Keywords: Information organization. Information visualization. Information retrieval systems.

\section{Título}

La contribución de la Organización y la Visualización de Información para Sistemas de Recuperación de Información

\section{Resumen}

Introducción: Tratando de proponer estrategias que mejoren la comprensión de la memoria almacenada en Sistemas de Recuperación de Información (SRIs), se considera en este trabajo, como alternativa, la adopción de los procedimientos de la Organización de la Información (IO) y la Visualización de la Información (VI). 

recuperação de informação

Objetivo: En este contexto, la investigación tiene como objetivo identificar y analizar la relación entre los aspectos de Ol y VI desde el punto de vista de la contribución de las dos áreas para los SRIs con el fin de mejorar la comprensión de la memoria almacenada por ellos, por qué la investigación se caracteriza por ser exploratoria y bibliográfica.

Metodología: El enfoque metodológico utilizado para llevar a cabo la envestigación consiste en levantamiento bibliográfico, para la obtención de bases científicas para una mejor comprensión y análisis de cada área, levantamiento de sistemas que hacen uso de interfaz de $\mathrm{VI}$ en su estructura, así como el análisis de los elementos de Ol necesarios para la organización y representación de los contenido a transmitirse en estructuras visuales.

Resultados: De esta forma es evidente el vínculo entre las áreas dirigida cuando se aplica en sistemas que tienen que ver con la recuperación de información.

Conclusiones: Por lo tanto, se concluyó que el $\mathrm{Ol}$ y $\mathrm{VI}$ pueden lograr resultados prometedores si trabajan en conjunto para optimizar la recuperación de la memoria almacenada en los SRIs.

Palabras clave: Organización de la información. Visualización de la información. Sistemas de recuperación de información.

Recebido em: 22.04.2014

Aceito em: 04.06.2015 